\title{
DROPLET AND BUBBLE NUCLEATION MODELED BY DENSITY GRADIENT THEORY - CUBIC EQUATION OF STATE VERSUS SAFT MODEL
}

\begin{abstract}
Václav VINŠ ${ }^{1}$, Jan HRUBÝ ${ }^{1}$, Barbora PLANKOVÁ ${ }^{1,2}$
Abstract: The study presents some preliminary results of the density gradient theory (GT) combined with two different equations of state (EoS): the classical cubic equation by van der Waals and a recent approach based on the statistical associating fluid theory (SAFT), namely its perturbed-chain (PC) modification. The results showed that the cubic EoS predicted for a given surface tension the density profile with a noticeable defect. Bulk densities predicted by the cubic EoS differed as much as by $100 \%$ from the reference data. On the other hand, the PC-SAFT EoS provided accurate results for density profile and both bulk densities in the large range of temperatures. It has been shown that PC-SAFT is a promising tool for accurate modeling of nucleation using the GT. Besides the basic case of a planar phase interface, the spherical interface was analyzed to model a critical cluster occurring either for nucleation of droplets (condensation) or bubbles (boiling, cavitation). However, the general solution for the spherical interface will require some more attention due to its numerical difficulty.
\end{abstract}

\section{INTRODUCTION}

Droplet and bubble nucleation plays an important role both in environmental processes (meteorology) and in many engineering applications (e.g. turbomachinery, evaporative cooling, and vapor compression refrigeration). Nucleation can be modeled with the Classical Nucleation Theory (CNT) [1]. CNT neglects the fact that the thickness of the vapor-liquid interface is comparable with the radius of the critical cluster (nano-droplet). To overcome this limitation, another approach was used in this study, namely the CahnHilliard density Gradient Theory (GT) [2-5]. The GT provides a general expression for the Helmholtz energy density of an inhomogeneous system. In combination with a convenient equation of state (EoS), the GT determines both the surface tension and the density profiles of the phase interface. The GT has been mostly used with a cubic EoS the van der Waals EoS [6] or the Peng-Robinson EoS $[7,8]$. Unlike other semi-empirical EoSs such as Benedict-Webb-Rubin [9] or Wagner's type [10], cubic EoS is based on a physical, though simplified, foundations. Consequently, a cubic EoS provides a qualitatively correct prediction also in the metastable region as it includes so called van der Waals loop. However, cubic EoSs have in general significant limitations in the accuracy of the predicted thermodynamic properties. Moreover, their application to complex mixtures and associating fluids such as water or alcohols is quite problematic.

\footnotetext{
1 Institute of Thermomechanics AS CR, v. v. i., Dolejškova 1402/5, 18200 Prague 8, Czech Republic (vins.vaclav@seznam.cz, hruby@it.cas.cz, barbora.plankova@gmail.com)

${ }^{2}$ Czech Technical University in Prague, Faculty of Nuclear Sciences and Physical Engineering, Břehová 7, 11519 Prague 1, Czech Republic
} 


\section{SAFT EquATION OF STATE}

A new physically-based EoS has been introduced by Chapman et al. [11,12] approximately twenty years ago. This new EoS, based on Statistical Associating Fluid Theory (SAFT), combines different intermolecular forces, e.g., hydrogen bonding, covalent bonding, Coulombic forces, for both spherical molecules and long non-spherical molecules. Variations of SAFT EoS (original SAFT [11], soft-SAFT [13], PC-SAFT [14], etc.) are successfully employed in modeling thermodynamic properties and phase equilibria of various substances and their complex mixtures. Thanks to the fact that SAFT EoS simulates directly the molecular structure of the fluids, it allows modeling also the metastable region, and, consequently, it can replace the cubic EoSs in the GT.

In this study, we implemented two different EoSs into the GT - the classical cubic EoS by van der Waals and the Perturbed Chain (PC) modification of the SAFT model [14]. Main aim of this study was to compare the predictive ability of both EoSs combined with the GT.

Table 1. Molar mass, critical temperature and pressure, and PC-SAFT parameters of considered substances

\begin{tabular}{lccccccc}
\hline Substance & $\mathbf{M}\left[\mathbf{k g} \cdot \mathbf{m o l}^{-\mathbf{1}} \mathbf{]}\right.$ & $\boldsymbol{T}_{\mathbf{c}}[\mathbf{K}]$ & $\left.\boldsymbol{p}_{\mathbf{c}} \mathbf{[ b a r}\right]$ & $\mathbf{m}[-]$ & $\mathbf{s}[\AA]$ & $\boldsymbol{\varepsilon} / \boldsymbol{k}_{\mathbf{B}}[\mathbf{K}]$ & Reference \\
\hline methane & 0.0160425 & 190.564 & 45.99 & 1.0000 & 3.7039 & 150.03 & {$[14]$} \\
propane & 0.0440956 & 369.825 & 42.48 & 2.0018 & 3.6184 & 208.11 & {$[14]$} \\
butane & 0.0581222 & 425.125 & 37.96 & 2.3421 & 3.7024 & 222.38 & {$[14]$} \\
n-heptane & 0.1002019 & 540.130 & 27.36 & 3.4832 & 3.8049 & 238.40 & {$[14]$} \\
n-nonane & 0.1282551 & 594.550 & 22.81 & 4.2073 & 3.8448 & 244.51 & {$[14]$} \\
n-decane & 0.1422817 & 617.700 & 21.03 & 4.6632 & 3.8384 & 243.87 & {$[14]$} \\
isobutane & 0.0581222 & 407.810 & 36.29 & 2.2616 & 3.7574 & 216.53 & {$[14]$} \\
carbon dioxide & 0.0440095 & 304.128 & 73.77 & 2.0727 & 2.7852 & 169.21 & {$[14]$} \\
R22 & 0.0864681 & 369.295 & 49.90 & 2.4746 & 3.1331 & 189.03 & our values \\
\hline
\end{tabular}

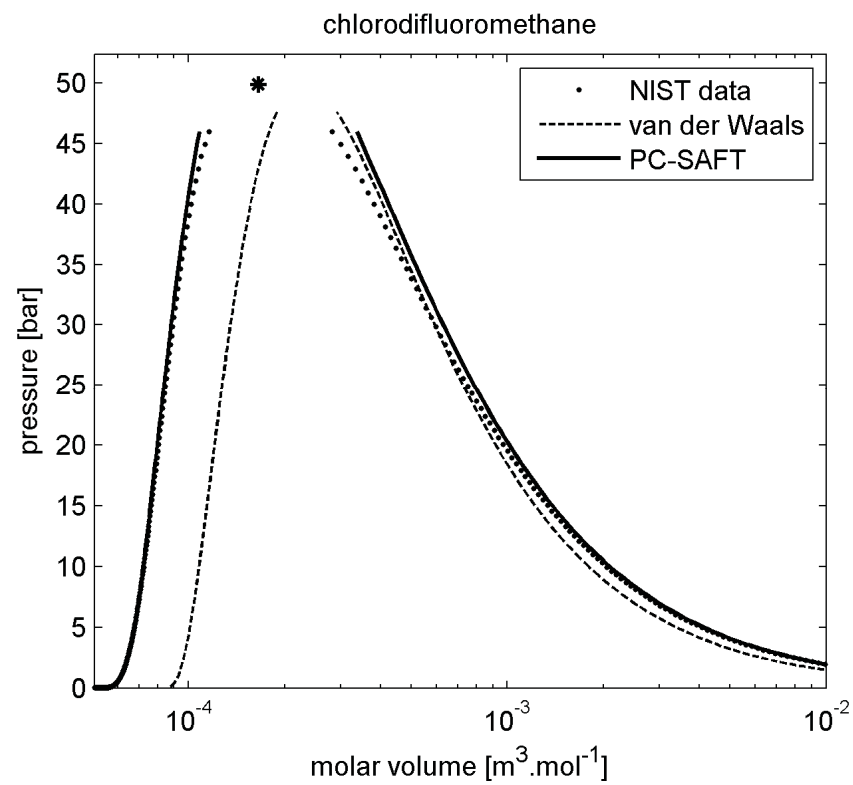

Figure 1. $p-V$ diagram of R22, comparison of NIST data with van der Waals EoS and PC-SAFT EoS 
The PC-SAFT model uses a description of non-associating molecules by three temperature independent parameters: the number of segments per chain $m$, the segment diameter $s$, and the segment energy parameter $\varepsilon$. Table 1 summarizes the PCSAFT parameters for nine substances considered in this work. The PC-SAFT parameters for refrigerant R22 (chlorodifluoromethane) had to be derived in a similar way as in our previous study [15], since no molecular parameters were found in the literature for this substance. Saturation pressure and density of saturated liquid were correlated to the data for R22 available at the NIST Webbook of chemistry [16].

Figure 1 compares NIST data for R22 with the predictions of van der Waals EoS and PCSAFT model. As can be seen, the van der Waals EoS interprets the saturation curves only roughly. The density of saturated liquid predicted by van der Waals EoS differs by more than $50 \%$ from the NIST data. On the other hand, PC-SAFT is in quite good agreement with the NIST data both on the vapor and liquid side. The PC-SAFT deviates from the NIST data only in the region close to the critical point (marked with star), since the molecular parameters were correlated only up to the maximum reduced temperature $T / T_{c}=0.95$.

\section{Density Gradient Theory}

The density gradient theory has been described by many authors; for more detail see for instance works of Cahn and Hilliard [2-4] or Davis and Scriven [5]. We provide only rough description of the GT in this work.

Cahn and Hilliard [2] considered the molecular gradients in the phase interface being much smaller than the intermolecular distance. In such a case, the Helmholtz free energy in a region of non-uniform density depends on local density and its local derivatives. Considering the molar density $\rho$ and density gradients $\nabla \rho$ as independent variables, the Helmholtz free energy density $f$ in a non-uniform region could be expanded as a Taylor series around the homogeneous state.

$$
F=\int_{V} f \mathrm{~d} V=\int_{V}\left[f_{0}(\rho)+\sum_{i, j} \frac{1}{2} c_{i j}(\rho) \nabla \rho_{i} \nabla \rho_{j}+\ldots\right] \mathrm{d} V
$$

Terms of the third and higher order of the Taylor series were neglected. The Helmholtz free energy density $f$ of a volume of non-uniform solution can be interpreted as sum of two contributions: the Helmholtz free energy the volume would have in a homogeneous fluid $f_{0}(\rho)$ and a corrective term cited also as a "gradient energy" which is function of the local density gradients. Coefficients $c_{i j}$ in the corrective term are called the influence parameters. They describe dependence of the density gradients on the local deviations of the chemical potentials and carry the information about the molecular structure of the phase interface. By minimizing the free energy $F$, the interfacial density profile must satisfy the following Euler-Lagrange equation:

$$
\sum_{j} \nabla\left(c_{i j} \nabla \rho_{j}\right)-\frac{1}{2} \sum_{j, k} \frac{\partial c_{j k}}{\partial \rho_{i}} \nabla \rho_{j} \nabla \rho_{k}=\frac{\partial \omega}{\partial \rho_{i}} .
$$

In equation (2), $\omega$ marks the grand-potential density defined as follows:

$$
\omega(\rho)=f_{0}(\rho)-\sum_{i} \rho_{i}\left(\frac{\mathrm{d} f_{0}}{\mathrm{~d} \rho_{i}}\right)_{T, V, \rho_{j}}=f_{0}(\rho)-\sum_{i} \rho_{i} \mu_{i}^{0} .
$$


For a planar phase interface between two bulk phases, which is the case considered further in this study, the gradients $\nabla \rho_{i}$ in equation (2) can be replaced by $\mathrm{d} \rho_{i} / \mathrm{d} z$. In most cases, the density-dependence of the influence parameter can be neglected and equation (2) obtains following simplified form:

$$
\sum_{j} c_{i j} \frac{\mathrm{d}^{2} \rho_{j}}{\mathrm{~d} z^{2}}=\frac{\mathrm{d} \omega}{\mathrm{d} \rho_{i}},
$$

with $z$ denoting the direction perpendicular to the phase interface. Multiplying equation (4) by $\mathrm{d} \rho_{i} / \mathrm{d} z$, summing over $i$ and integrating gives:

$$
\sum_{i, j} \frac{1}{2} c_{i j} \frac{\mathrm{d} \rho_{j}}{\mathrm{~d} z} \frac{\mathrm{d} \rho_{i}}{\mathrm{~d} z}=\omega(\rho)-\omega_{0},
$$

where $\omega^{0}$ is the integration constant corresponding to the grand-potential density of a homogeneous phase, which equals the negative value of the equilibrium pressure $\omega^{0}=-p^{0}$.

Taking into account definition of the gradient theoretical tension [5]

$$
\sigma=\sum_{i, j} \int_{-\infty}^{\infty} c_{i j} \frac{\mathrm{d} \rho_{i}}{\mathrm{~d} z} \frac{\mathrm{d} \rho_{j}}{\mathrm{~d} z} \mathrm{~d} z
$$

the surface tension of a multi-component mixture can be derived from equations ( 3 ) and (5) in the following manner:

$$
\sigma=2 \int_{-\infty}^{\infty}\left[f_{0}(\rho)-\sum_{i} \rho_{i} \mu_{i}^{0}+p^{0}\right] \mathrm{d} z=2 \int_{-\infty}^{\infty}\left[\omega(\rho)-\omega^{0}\right] \mathrm{d} z=2 \int_{-\infty}^{\infty} \Delta \omega(\rho) \mathrm{d} z .
$$

Equation (7) can be considered as the main result of the GT applied on the planar phase interface. It allows predicting the surface tension both for pure fluids and for complex mixtures once a convenient EoS is available.

\section{Planar Phase Interface of a Pure Fluid}

Let us consider the planar phase interface of a pure non-associating fluid. In such a case, equation (5) becomes following form:

$$
\frac{1}{2} c\left(\frac{\mathrm{d} \rho}{\mathrm{d} z}\right)^{2}=\omega(\rho)-\omega^{0}=\Delta \omega,
$$

which can be simply modified to obtain the relation between $\mathrm{d} \rho$ and $\mathrm{d} z$ :

$$
\mathrm{d} z=\sqrt{\frac{c}{2 \Delta \omega}} \mathrm{d} \rho \text {. }
$$

Considering, for instance, following boundary conditions $\rho(z \rightarrow-\infty)=\rho_{V}$ and $\rho(z \rightarrow \infty)=\rho_{L}$, the surface tension of a pure fluid can be expressed as:

$$
\sigma=2 \int_{-\infty}^{\infty} \Delta \omega(\rho) \mathrm{d} z=\int_{\rho_{V}}^{\rho_{L}} \sqrt{2 c \Delta \omega(\rho)} \mathrm{d} \rho .
$$

Equation (10) defines the surface tension as a function of the excess grand-potential density $\Delta \omega$ - derived from the available EoS - and influence parameter $c$. In further calculations, we assume the influence parameter independent of temperature, i.e. $c$ is treated as a constant. This simplification allows us to predict the surface tension over a 
wide range of temperatures once the influence parameter is known. Table 2 summarizes values of the influence parameters for all substances considered in this work. Values for $c$ were derived from equation (10) by correlating the available experimental data for surface tension.

Table 2. Estimated values of the influence parameter for PC-SAFT with temperature ranges of the surface tension experimental data

\begin{tabular}{lcccc}
\hline Substance & $\boldsymbol{c}\left[\mathbf{J} \cdot \mathbf{m}^{\mathbf{5}} \cdot \mathbf{m o l}^{-\mathbf{2}} \mathbf{]}\right.$ & $\boldsymbol{T}_{\mathbf{m i n}}[\mathbf{K}]$ & $\boldsymbol{T}_{\boldsymbol{m a x}}[\mathbf{K}]$ & Reference for $\boldsymbol{\sigma}$ \\
\hline methane & $1.956038 \mathrm{E}-20$ & 90.0 & 115.0 & {$[17,18]$} \\
propane & $1.006459 \mathrm{E}-19$ & 183.2 & 360.6 & {$[17,23-25]$} \\
butane & $1.827412 \mathrm{E}-19$ & 203.2 & 318.5 & {$[17,22,23]$} \\
n-heptane & $4.590055 \mathrm{E}-19$ & 183.2 & 522.2 & {$[20,21,26,27]$} \\
n-nonane & $7.716970 \mathrm{E}-19$ & 273.2 & 333.2 & {$[20]$} \\
n-decane & $9.673225 \mathrm{E}-19$ & 273.2 & 343.2 & {$[20,27]$} \\
isobutane & $1.609301 \mathrm{E}-19$ & 236.9 & 396.5 & {$[22,25]$} \\
carbon dioxide & $1.863571 \mathrm{E}-20$ & 243.2 & 297.9 & {$[17,19]$} \\
R22 & $6.340054 \mathrm{E}-20$ & 120.0 & 365.0 & {$[16]-$ NIST data } \\
\hline
\end{tabular}

Figure 2 shows temperature dependence of the surface tension for propane. The experimental data [17,23-25] are compared with the data from NIST Webbook of chemistry [16] and with the GT predictions. As can be seen, the GT in combination with PC-SAFT provides quite good results over a wide range of temperature compared to the van der Waals EoS. The GT + PC-SAFT agrees also with the NIST data. Set of five experimental points lying below other data was taken from the work of Jasper [17]. This data is most likely corrupted, since it disagrees both with other experimental data and GT predictions.

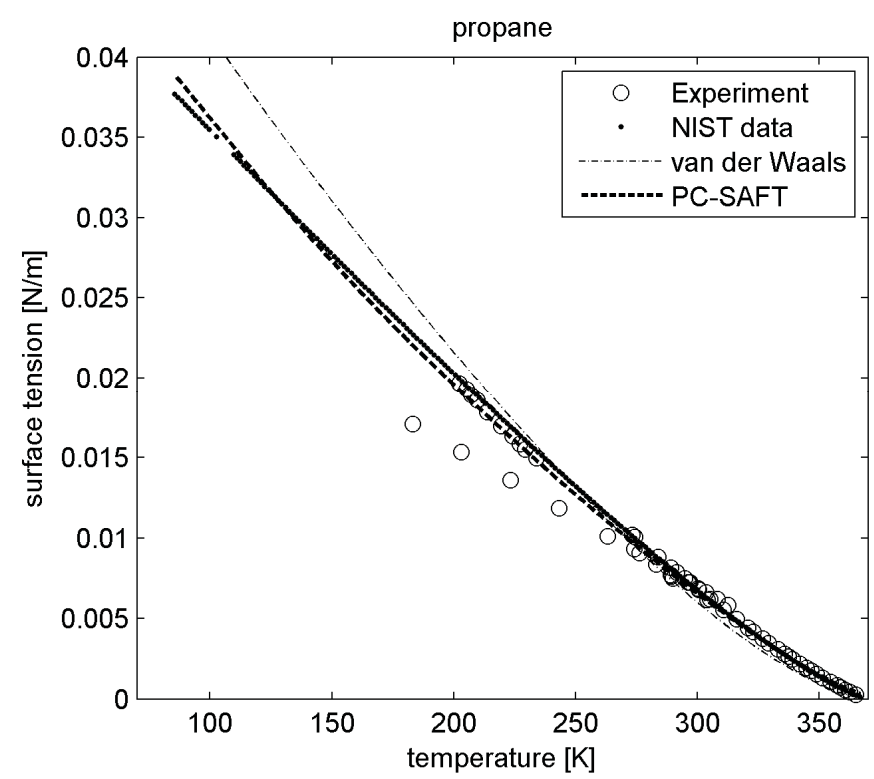

Figure 2. Surface tension for propane. Experimental data versus NIST data, van der Waals EoS and PC-SAFT EOS $\left(c_{\mathrm{vdW}}=1.1521 \cdot 10^{-18} \mathrm{~J} \cdot \mathrm{m}^{5} \cdot \mathrm{mol}^{-2}\right)$

Another example of surface tension predicted by the GT is shown in Figure 3 for $\mathrm{CO}_{2}$. The GT predictions are compared with the experimental data of Jasper [17] and Rathjen W. and Straub [19] and with the NIST data [16]. The GT in combination with PC-SAFT 
provides quite good results compared to van der Waals EoS again. However at the temperatures below $260 \mathrm{~K}$, the predicted surface tension is slightly underestimated. This can be caused by the fact that the influence parameter was correlated to the surface tension experimental data at relatively high temperatures around $280 \mathrm{~K}$.

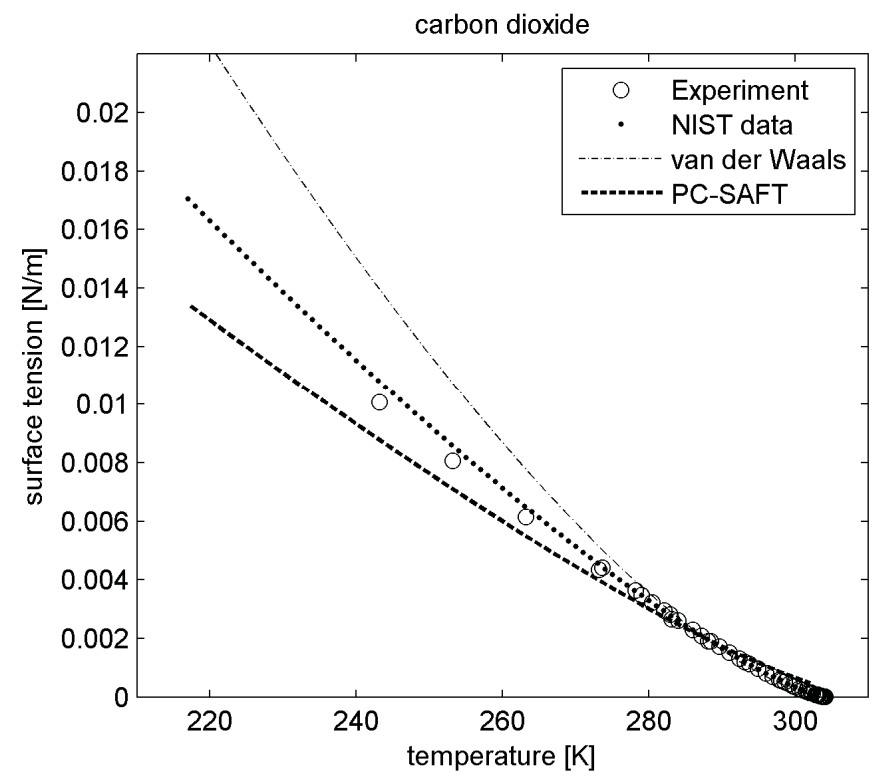

Figure 3. Surface tension for $\mathrm{CO}_{2}$. Experimental data versus NIST data, van der Waals EoS and PC-SAFT EOS $\left(c_{\mathrm{vdW}}=6.7915 \cdot 10^{-19} \mathrm{~J} \cdot \mathrm{m}^{5} \cdot \mathrm{mol}^{-2}\right)$

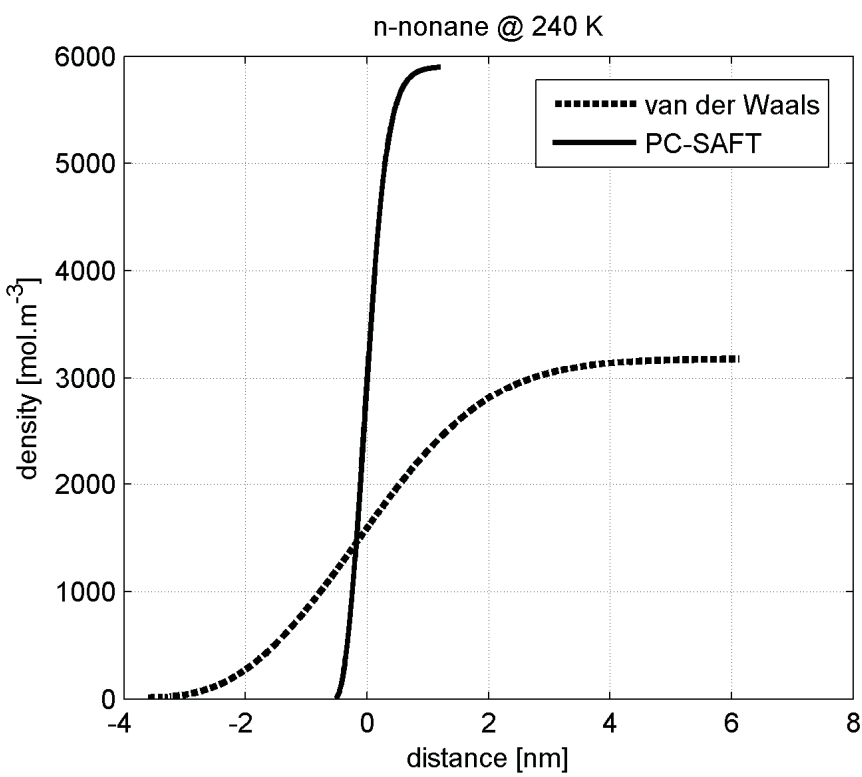

Figure 4. Planar density profiles predicted by the GT combined with the van der Waals EoS and with PC-SAFT EoS for $n$-nonane at $240 \mathrm{~K}$ $\left(c_{\mathrm{vdw}}=1.5527 \cdot 10^{-17} \mathrm{~J} \cdot \mathrm{m}^{5} \cdot \mathrm{mol}^{-2}\right)$

The density profile over a planar phase interface can be calculated from integrated equation (9) as follows:

$$
z-z_{0}=\int_{\rho_{0}}^{\rho} \sqrt{\frac{c}{2 \Delta \omega}} \mathrm{d} \rho .
$$


Figure 4 compares predicted density profiles of a planar phase interface for $n$-nonane at temperature $240 \mathrm{~K}$. It is clear that both GT predictions show significant difference. The liquid bulk density predicted by the van der Waals EoS is almost twice smaller than its correct value $5925 \mathrm{~mol} . \mathrm{m}^{-3}$. On the other hand, PC-SAFT predicts the profile quite well.

\section{Spherical Phase Interface}

Besides basic case of a planar phase interface, we use the GT on a spherical interface, i.e. on droplets and bubbles of a finite diameter. This case is important for modeling the initial period of phase transitions - nucleation of droplets or bubbles during condensation or evaporation, respectively.

Let us focus on a closed one component system both in liquid and vapor bulk phases of a same temperature and chemical potential. However in such a case, the pressure can vary along the phase interface and the system is not in a thermodynamic equilibrium.

From the infinite number of possible sizes of the nucleus (droplet or bubble) at a given temperature and supersaturation, we consider so called critical size defined by critical radius. Critical radius has in principle the same probability of growing or reducing.

Using the GT, a second order differential equation can be derived from equation (2) for a spherical phase interface:

$$
-\frac{\mathrm{d}^{2} \rho(r)}{\mathrm{d} r^{2}}-\frac{2}{r} \frac{\mathrm{d} \rho(r)}{\mathrm{d} r}+\frac{\Delta \mu(\rho(r))}{c}=0 .
$$

In equation (12), variable $r$ marks the radial coordinate and $\Delta \mu$ is the difference of the chemical potential varying with local density $\mu(\rho)$ and the constant chemical potential of the bulk phase $\mu^{0}$. We consider following boundary conditions for equation (12):

$$
\frac{\mathrm{d} \rho(0)}{\mathrm{d} r}=0, \rho\left(r_{c}\right)=\rho_{B},
$$

stating that in the centre of a nucleus the density change equals zero, and that beyond a sufficiently large radius $r_{c}$ there will be bulk density $\rho_{B}$ (index $B=V$ for a droplet and $B=L$ for a bubble).

The boundary problem defined by equations (12) and (13) is solved numerically using the shooting method when the density in the nucleus centre $\rho(0)$ is iteratively estimated. This transforms the boundary problem into the initial value problem, which can be gradually solved by Runge-Kutta method. In such a case, the entire problem reduces to initial value estimation.

The problem of initial value estimation can be further simplified. In the centre of a nucleus and at the radius of a cluster, i.e. at the onset of the bulk phase, equation (12) can be linearized. Using Bessel functions [17], the problem can be computed analytically in both boundary regions. The analytical solution is more accurate here as it computes the density directly and avoids cumulation of numerical errors.

It turns out that there are three different solutions of equation (12): (a) the solution corresponding to pure phase with a constant density $(\rho(r)=\rho(0))$, (b) density profile solution fulfilling condition (13), and (c) a physically incorrect density profile. Case (a) is from the mathematical point of view uninteresting and can be simply avoided. Unlike the third case, case (b) representing physically correct solution appeared to be numerically unstable. This fact resulted in the main complication of the algorithm. The problem of case (b) is that after reaching the bulk density $\rho_{B}$, the density profile starts to oscillate 
around numerically stable solution (c). This makes the second condition in equation (13) almost impossible to achieve. Moreover for higher values of the initial density, the Runge-Kutta method fails and provides solution in the field of complex numbers. Last but not least, it is not possible to solve larger nuclei, i.e. at low supersaturation, since the algorithm reaches maximum accuracy of the computing code.

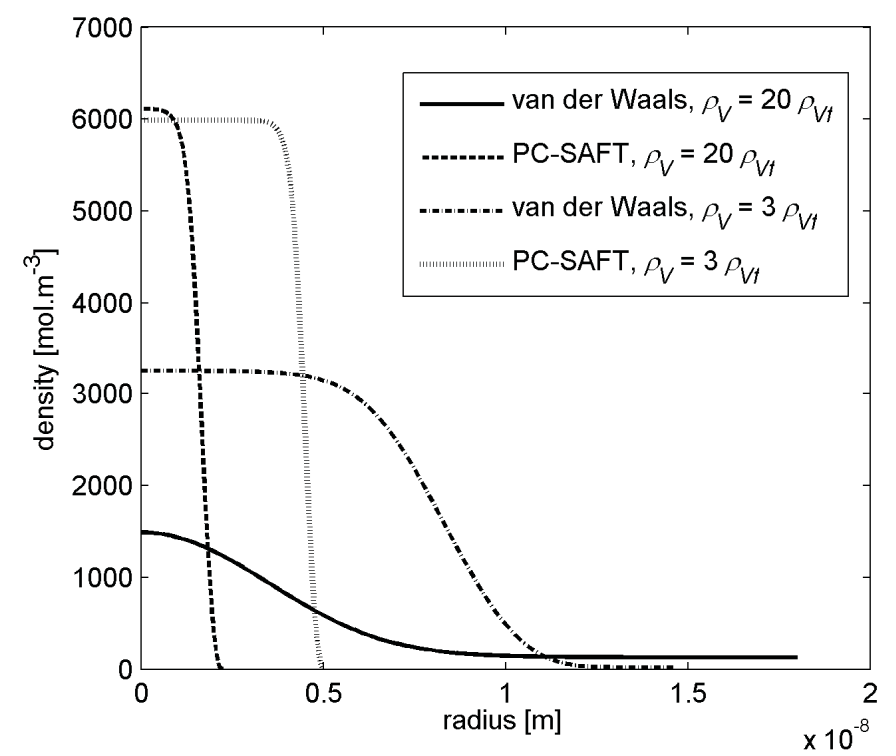

Figure 5. Density profiles over spherical phase interface predicted by the GT combined with the van der Waals EoS and with PC-SAFT EoS for n-nonane at $240 \mathrm{~K}\left(c_{\mathrm{vdw}}=1.5527 \cdot 10^{-17} \mathrm{~J} \cdot \mathrm{m}^{5} \cdot \mathrm{mol}^{-2}\right)$

To deal with all the problems mentioned above we have tried several other modifications of the algorithm. The secant method has been used to estimate the initial value of density in the shooting method. However, this approach did not work well. Currently we are using the bisection method, which provides the most promising results until now. Figure 5 presents an example of the density profiles along spherical phase interface calculated with van der Waals EoS and PC-SAFT model. As can be seen, the droplets' diameter increases with lower supersaturation, i.e. lower bulk density $\rho_{V}$.

\section{SUMMARY}

Two different EoSs were implemented into the GT. The results showed that the PC-SAFT model provides quite accurate predictions of both the planar phase interface and surface tension compared to classical van der Waals EoS. Values of a constant influence parameter were evaluated for nine selected substances, which will be used in subsequent modeling of the spherical phase interface and planar phase interface of a binary mixture. Preliminary results of the model predicting critical radius of a spherical phase interface, i.e. droplet and bubble, were presented. The model works well for higher temperatures and high level of supersaturation. However, some numerical problems still occur at lower temperatures. Our main task is to improve the convergence of the algorithm even for large nuclei.

Another part of our contemporary activity is connected with modeling the planar phase interface for a binary mixture. The GT combined with PC-SAFT EoS is being modified for 
binary mixtures of non-associating fluids now. The cross-influence parameters $c_{i j}$ may be estimated by simple mixing rule $c_{i j}=\sqrt{c_{i i} c_{j j}}$.

\section{NOMENCLATURE}

$C$... influence parameter []. $\left.\mathrm{m}^{5} \cdot \mathrm{mol}^{-2}\right]$

$F$... Helmholtz free energy [J]

$f$... Helmholtz free energy density [ $\left.{ }^{-\mathrm{m}^{-3}}\right]$

$k_{\mathrm{B}} \ldots$ Boltzmann constant [ $\left.\mathrm{J}^{\mathrm{K}} \mathrm{K}^{-1}\right]$

$M$... molar mass $\left[\mathrm{kg} \cdot \mathrm{mol}^{-1}\right]$

$m$... segment number [-]

$p$... pressure $[\mathrm{Pa}]$

s... segment diameter $\left[\AA=10^{-10} \mathrm{~m}\right]$

$T$... temperature $[\mathrm{K}]$

$V \quad$... volume $\left[\mathrm{m}^{3}\right]$

$\omega \ldots$ grand-potential density $\left[\mathrm{J} \cdot \mathrm{m}^{-3}\right]$

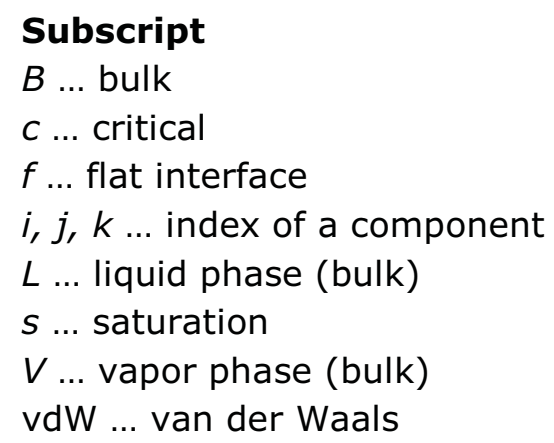

\section{Greek letters}

$\varepsilon$... segment energy parameter [J]

$\rho$... density [mol. $\mathrm{m}^{-3}$ ]

$\mu$... chemical potential []$\left.\cdot \mathrm{mol}^{-1}\right]$

$\sigma \ldots$ surface tension $\left[\mathrm{N} . \mathrm{m}^{-1}\right]$

\section{Superscript}

0 ... homogeneous

\section{References}

[1] Vehkamäki H.: Classical Nucleation Theory in Multicomponent Systems, Springer-Verlag Berlin Heidelberg, 2006, ISBN-10 3-540-29213-6

[2] Cahn J.W. and Hilliard J.E.: Free energy of a nonuniform system I. Interfacial free energy, J. Chem. Phys. 28 (2), 1958, 258-267

[3] Cahn J.W.: Free energy of a nonuniform system II. Thermodynamics basis, J. Chem. Phys. 30 (5), 1959, 1121-1124

[4] Cahn J.W. and Hilliard J.E.: Free energy of a nonuniform system III. Nucleation in a two-component incompressible fluid, J. Chem. Phys. 31 (3), 1959, 688-699

[5] Davis H. T. and Scriven L. E.: Stress and Structure in Fluid Interfaces, Advances in Chemical Physics 49, 1982, 357-454

[6] Carey B.S., Scriven, L.E., Davis, H.T.: Gradient theories of fluid interfacial stress and structure, J. Chem. Phys. 69, 1978, 5040-5049

[7] Zuo Y.X. and Stenby E.H.: Calculation of interfacial tensions with gradient theory, Fluid Phase Equilib. 132, 1997, 139-158

[8] Hrubý J., Labetski D.G., van Dongen M.E.H.: Gradient theory computation of the radius-dependent surface tension and nucleation rate for n-nonane clusters, $J$. Chem. Phys. 127 (16), 2007

[9] Benedict M., Webb G.B., Rubin L.C.: An empirical equation for thermodynamic properties of light hydrocarbons and their mixtures I. Methane, ethane, propane and n-butane, J. Chem. Phys. 8, 1940, 334

[10] Setzmann U. and Wagner W.: A new method for optimizing the structure of thermodynamic correlation equations, Int. J. Thermophys. 10, 1989, 1103-1126

[11] Chapman W.G., Gubbins K.E., Jackson G., Radosz M.: SAFT-Equation-of-state solution model for associating fluids, Fluid Phase Equilib. 52, 1989, 31-38 
[12] Chapman W.G., Gubbins K.E., Jackson G., Radosz M.: New reference equation of state for associating liquids, Ind. Eng. Chem. Res. 29, 1990, 1709-1721

[13] Blas F.J. and Vega L.F.: Prediction of binary and ternary diagrams using the statistical associating fluid theory (SAFT) equation of state, Ind. Eng. Chem. Res. 37, 1998, 660-674

[14] Gross J. and Sadowski G.: Perturbed-chain SAFT: An equation of state based on a perturbation theory for chain molecules, Ind. Eng. Chem. Res. 40, 2001, $1244-1260$

[15] Vinš V. and Vacek V.: Effect of gas impurities on the throttling process of fluorocarbon refrigerants: Estimation of the Henry's law constant, J. Chem. Eng. Data 54 (9), 2009, 2395-2403

[16] NIST WebBook Chemistry, August 2010, http://webbook.nist.gov/chemistry/

[17] Jasper J.J.: The surface tension of pure liquid compounds, J. Phys. Chem. Ref. Data 1, 1972, 841-1009

[18] Fuks S. and Bellemans A.: The surface tension of krypton, methane and their mixtures, Physica 32, 1966, 594-602

[19] Rathjen W. and Straub J.: Temperature dependence of surface tension, coexistence curve, and vapor pressure of $\mathrm{CO}_{2}, \mathrm{CClF}_{2}, \mathrm{CBrF}_{3}$, and $\mathrm{SF}_{6}$, Heat transfer in boiling, Eds.: Hahne E. and Grigull U., Academic Press 1977, 425-451

[20] Jasper J.J., Kerr R.E., Gregorich F.: The orthobaric surface tensions and thermodynamic properties of the liquid surfaces of the n-alkanes, $\mathrm{C}_{5}-\mathrm{C}_{18}, \mathrm{~J} . \mathrm{Am}$. Chem. Soc. 75, 1953, 5252-5254

[21] Grigoryev B.A., Nemzer B.V., Kurumov D.S., Sengers J.V.: Surface tension of normal pentane, hexane, heptane and octane, Int. J. Thermophys. 13 (3), 1992, 453-464

[22] Coffin C.C. and Maass O.: The preparation and physical properties of alpha-, beta- and gamma-butylene and normal and isobutene, J. Am. Chem. Soc. 50, $1928,1427-1437$

[23] Katz D.L. and Saltman W.: Surface tension of hydrocarbons, Ind. Eng. Chem. 31, 1939, 91-94

[24] Maass O. and Wright $\mathrm{H}$.: Some physical properties of hydrocarbons containing two and three carbon atoms, J. Am. Chem. Soc. 43, 1921, 1098-1111

[25] Baidakov V.G. and Sulla I.I.: Pverchnostnoe natiazhenie propana i izobutana pri temperaturach, blizkich k kriticheskoi, Zhurnal Fizicheskoi Chimii LIX, 1985, 955-957

[26] Voliak L.D. and Andreeva L.P.: Issledovanie poverchnostvogo natiazenia ngeptana i n-oktana, Zhurnal Fiziceskoi Chimii XXXV, 1961, 1416-1417

[27] Rolo L.I., Caco A.I., Queimada A.J., Marrucho I.M., Coutinho J.A.P.: Surface tension of heptane, decane, hexadecane, eicosane, and some of their binary mixtures, J. Chem. Eng. Data 47, 2002, 1442-1445

[28] Abramowitz M. and Stengun I.A.: Handbook of mathematical functions with formulas, graphs, and mathematical tables, New York: Dover Publications, 1972, ISBN 978-0-486-61272-0

\section{ACKNOWLEDGEMENTS}

The project has been supported by grant IAA200760905 and by the Research Plan of the Institute of Thermomechanics AS ČR, v. v. i. No. AV0Z20760514. 